\title{
A TOPOLOGICAL APPROACH TO INFINITY IN PHYSICS
}

\author{
Arturo Tozzi \\ Center for Nonlinear Science, Department of Physics, University of North Texas, Denton, Texas 76203, USA \\ 1155 Union Circle, \#311427, Denton, TX 76203-5017 USA \\ tozziarturo@libero.it \\ Arturo.Tozzi@unt.edu
}

\author{
James F. Peters \\ Department of Electrical and Computer Engineering, University of Manitoba \\ 75A Chancellor's Circle \\ Winnipeg, MB R3T 5V6 Canada and \\ Department of Mathematics, Faculty of Arts and Sciences, Adiyaman University \\ 02040 Adiyaman, Turkey \\ James.Peters3@umanitoba.ca
}

\begin{abstract}
Physical measurements might display range values extending towards infinite. The occurrence of infinity in physical equations, such as the black hole singularities, is a troublesome issue that causes many theories to break down when assessing extreme events. Different methods, such as re-normalization, have been proposed to avoid detrimental infinity. Here a novel technique is proposed, based on physical geometrical considerations and the Alexander Horned sphere, that permits to undermine infinity in physical equations. In this unconventional approach, a continuous monodimensional line becomes an assembly of countless bidimensional lines that superimpose in quantifiable knots and bifurcations.
\end{abstract}

KEYWORDS: donut-like; infinity; paths; physical geometry; networks, Alexander horned sphere.

Excluding conformal infinity (Frauendiener, 2000), the occurrence of infinite results in physical formulas prevents experimental descriptions of real observables (Bergmann, 1989). For example, when assessing bodies with infinite gravitational mass and/or energy, equations become untreatable and lead to failures in theories. A standard example is the mathematical singularity of black holes, where the commonest solutions of the equations of general relativity allow for zero-size, finite mass distributions. This leads to infinite density, e.g., singularities where relativity breaks down. The problem becomes troublesome, if we think that infinity is hidden also in the most unlikely discipline, i.e., quantum mechanics. Despite quantum description is framed on the concept of indivisible elements, it must be stressed that its postulates dictate dynamics taking place inside a Hilbert space of infinite dimensions. In quantum mechanics, infinity runs out the door and comes back in the window. To make the problem more intricate, recent claims suggest that infinite sets cannot be reduced to finite sets (in technical terms, the Connes conjecture is refutable). Indeed, it seems that infinity cannot be reduced to finite, at least in the context of peculiar sets (Ji et al., 2020). The problem is worsened by the Mandelstamm-Tamm inequality for quantum systems (Mandelstam and Tamm, 1945), that dictates a general lower bound for the lifetime of all quantum states in terms of reasonable measures of uncertainty in energy, even if the standard deviation is infinite. Indeed, according to a formulation of the Heisenberg principle, energy and time can be related by the formula:

$\Delta \mathrm{E} \Delta \mathrm{T} \geq \frac{h}{4 \pi}$

where $\Delta \mathrm{E}$ is a non-infinite uncertainty in energy that is independent of time. This means that a significant change in energetic conformation may occur in a very short time interval. In turn, very short time intervals lead to huge fluctuations in energy which tend towards infinite values.

In sum, although infinity can be used in physics, scientists require for practical purposes the final results to be physically meaningful: e.g., in quantum field theory, infinities are treated through procedures such as renormalization ('tHooft G. 1971). Here we propose a fully novel, topologically-framed, approach to the issue of infinity in physical equations. 


\section{GEOMETRICAL CONCEPTS OF INFINITY}

The concept of infinity is strictly correlated with the long-standing geometrical problem concerning the line: is a line composed of indivisibles, or is it a infinitely divisible continuum? Throughout the centuries, many different answers have been provided to this painstaking question (Bell 2005). For example, Aristotle, Al Ghazali and Averroes stated that a line is not composed of atoms, but rather of parts divisible without an end (Aristotle 1980). Democritus, Leucippus and Epicurus maintained that a continuous line is composed of indivisible bodies. Pythagoras, Plato and Walter de Chatton (about 1323) claimed that a line is composed by an finite number of indivisibles, while others by an infinite number of indivisibles. In this latter group, Henry of Harclay (1275-1317) stated that a line is composed by an infinite number of immediately joined indivisibles, while the Bishop of Lincoln that it is composed by an infinite number of indivisibles which are mediate to one another. According to the more mathematical approach of Richard Kilvington (about 1302-1360), a continuum stands for infinite sets of integers containing infinite subsets with intrinsic and extrinsic quantifiable boundaries. Adam of Whodeham (death: 1358) suggested that one infinite can be greater than another. In turn, Bradwardine, outstripping the contemporary mathematical views, believed that a continuum is not composed of indivisible points, but of mutually joined parts, i.e., an infinite number of mediate indivisibles that are not points (Bradwardine 1328-1335). William of Ockham said that a continuum whose parts form a unity is distinct from a contiguum of juxtaposed things. In the following centuries, the issue was tackled by foremost Authors, including Isaac Barrow, Leibnitz, Guillame de L'Hópital, Euler, Berkeley. The continuum in terms of infinitesimal was then tackled by Bernard Bolzano, Augustin-Louis Cauchy, Karl Weierstrass, Richard Dedekind (Dedekind 1963). Different solutions were proposed, until Georg Cantor introduced the theory of transfinite numbers that led to the modern concept of abstract set (Cantor 1961). In Cantor's view, it was numbers, rather than geometric points, that are equipped with objective significance. Critical reactions were put forward by Brentano, Peirce, Poincaré (Poincaré 1946), Brouwer, Hermann Weyl. Apart from the most advanced contemporary theories -see Abram Robinson (1996), Bridges (1999), Lawvere (1980)-, in calculus and theory of function it is assumed that between any two rational points there are rational points. For every convergent series of such fractions there is just one limiting points: this collection of limiting points it termed continuous. In particular, in modern topology, a continuum is defined as a compact connected subset of a topological space.

Infinity as a straight line in an unusual historical geometrical approach. One of the most striking treatments of infinity is the one by the Cusa (de Cusa 1440), that allows to use mathematical and geometrical features to undertake physical infinity. Notice that de Cusa pictures the infinite as a straight line. Infinite circles or spheres can be described in terms of infinite lines: when the diameter becomes less curved in proportion to the increased circumference, then the maximum circumference, which cannot be greater, is minimally curved and therefore maximally straight. An infinite line is necessarily the straightest, so that no curvature is opposed. In the same way, every infinite manifold with positive curvature (an infinite triangle, an infinite square) can be described in terms of an infinite maximum line that si longest and straightest. Nor will this maximum manifold be composed of sides and angles; rather, the infinite line and angle are one and the same thing, so that the line is the angle, because the manifold is the line. In sum, an infinite line stands, according to de Cusa, for all that which is in the possibility of every finite line and manifold. Hence, a speculative inference can be made, namely, infinity is correlated with finite manifolds. In current scientific terms, de Cusa's account sound as follows: given a physical system described by progressively increasing curves on a positivecurvature manifold, the unwanted occurrence of a straight line (standing for infinite value in equations) can be erased by adding progressively decreasing curves on an opposite negative-curvature manifold. In sum, infinity can be expressed by a straight line with zero-curvature. This observation has been used in the mathematical treatment of physical finite systems whose equations lead to the unwanted infinity by Tozzi and Peters (2019), who employed geometrical procedures (Frankel T. 2011), Langevin equations (Langevin 1908), Levi-Civita and Ehresmann connections (LeviCivita1917; Ehresmann 1950).

\section{TOPOLOGY COMES INTO PLAY}

In this paragraph, we suggest a geometrically-framed method to solve the problem of infinity in physical systems. If one wants to assess finite physical measurements leading to infinity, one needs at first to consider finite mathematical figures and topological manifolds, together with their features and relations. Next, one must apply these relations in a projective way. Thirdly, one must thereafter, in a still more highly transformed way, apply the relations of these infinite figures to the general concept of mathematical infinity, which is altogether independent even of all figures and manifolds.

Alexander horned sphere: a toroidal phase space for both infinite and discrete lines. To make the description of a line that assesses quantitative values of both indivisible and divisible features, we propose to use a peculiar torus-like phase space, modified from the pathological object termed horned sphere (Alexander 1924). An Alexander horned sphere (AHS) is a topological structure made up of a countable union of compact sets. The mapping of AHS is 
homeomorphic with the ball $\mathrm{B}^{3}$, its boundary being a sphere (Figure 1A). AHS, together with its inside, stands for a topological 3-ball which is simply connected (i.e., every finite, simple closed curve can be shrunk to a point by a sequence of retraction mappings while staying inside). However, the outer complement of the solid is not simply connected and its fundamental group is not finitely generated. This provides an example of a wild embedding in $\mathrm{E}^{3}$ : the exterior is not simply connected (unlike the exterior of the usual round sphere). This means that a closed curve linking a torus in the above construction cannot be shrunk to a point without touching the horned sphere (Figures 1B-C). Because AHS displays an uncountable infinity of wild points which are the limits of the sequences, it has also been used to describe wild embeddings in quantum states (Asselmeyer-Maluga, 2018). Furthermore, the set of nonlocally flat points is a Cantor set. AHS displays branch points on the sphere (roughly, the "ends" of the horns), since any neighborhood of a limit contains a horned complex.

What matters in our framework is that the bifurcation paths approaching an infinite number are disjoined, i.e., no bifurcation is in touch with another on the opposite side (Figure 1C). To treat together both finite and infinite quantities, we require an AHS torus-like structure equipped with two different functional areas (Figure 2):

a) a circular section, on which indivisible features can be evaluated.

b) a branched section, equipped with ad libitum infinite bifurcations, on which continuum can be evaluated. This means that the continuum inside a one-dimensional line is treated in terms of superimposition of countless lines in two dimensions.
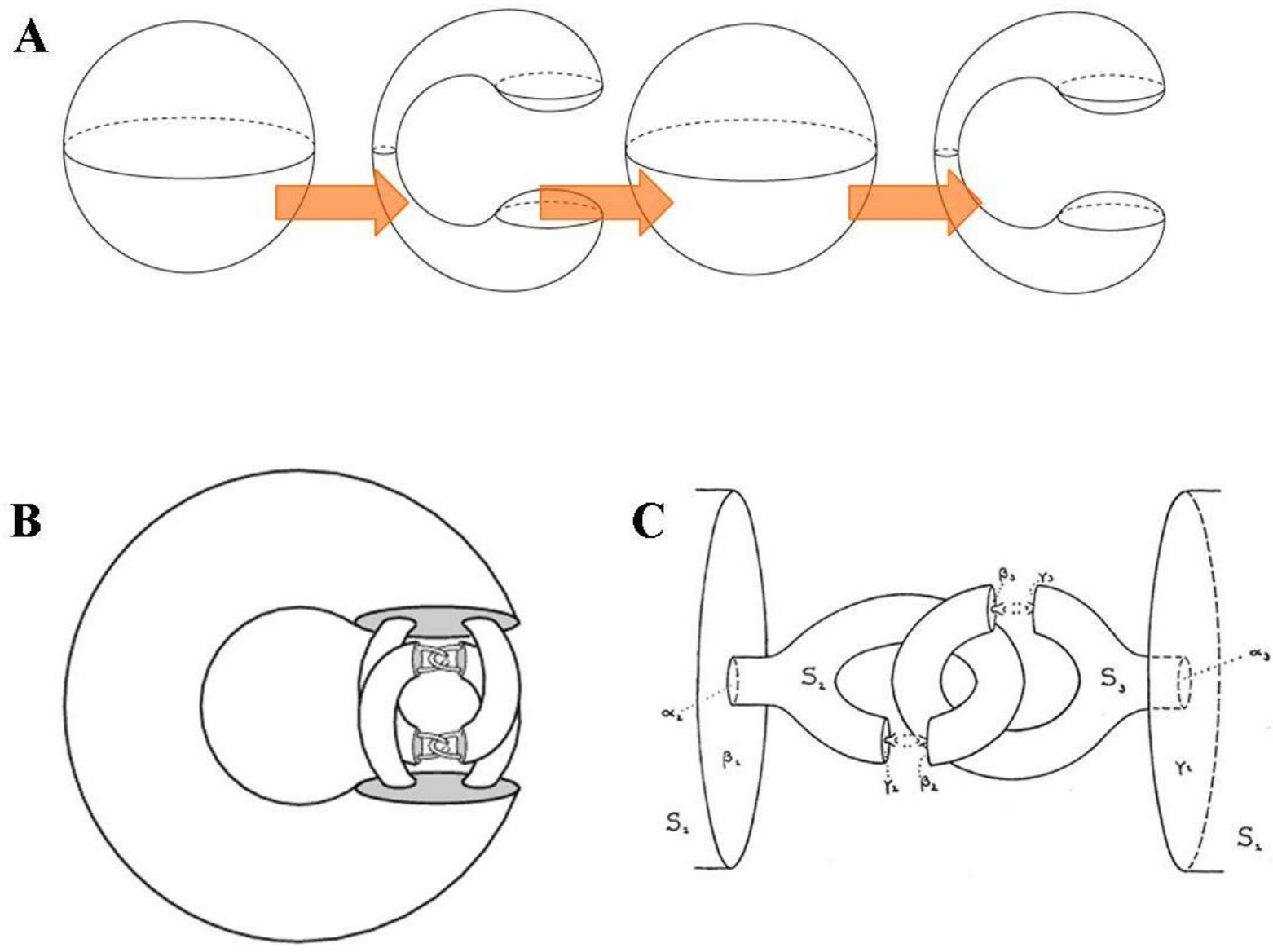

Figure 1. Alexander Horned sphere (AHS). Figure 1A: AHS can be built from a positive-curvature manifold with genus zero. Figures 1B-C: AHS at different magnifications. Figure 1C displays the original draw and nomenclature provided by Alexander (1924).. 
A

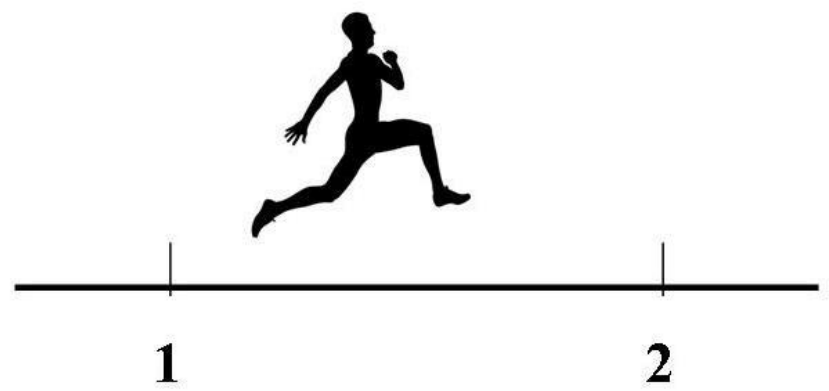

B

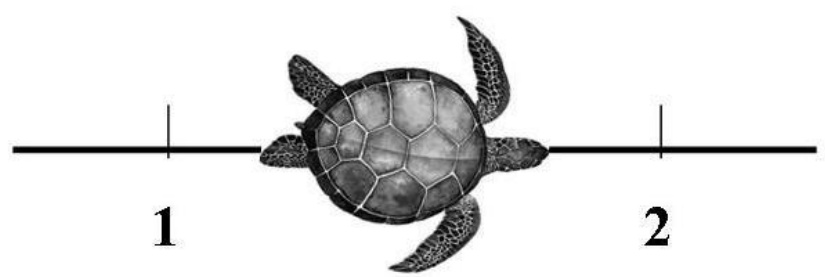

C

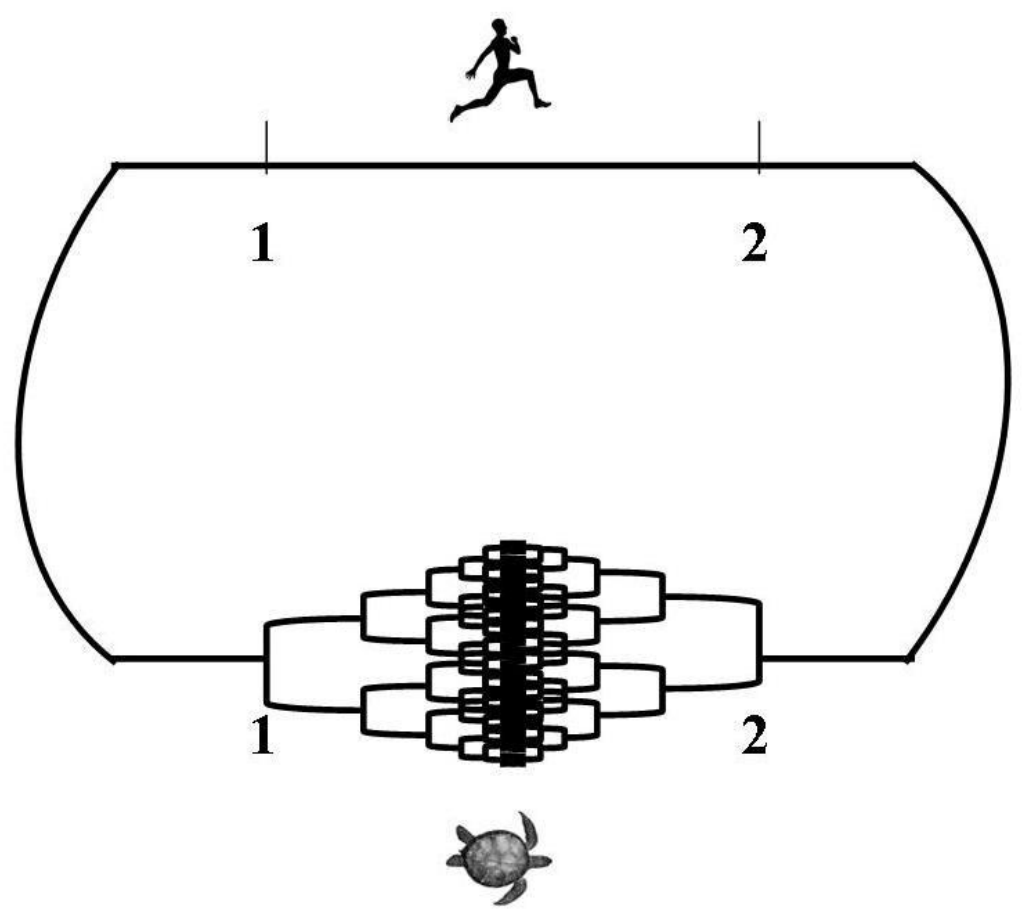

Figure 2. The geometric line in terms of divisible (2A) and undivisible (2A) components. Figure $\mathbf{2 C}$ depicts the two sides of an Alexander horn torus, encompassing both divisible (upper part) and undivisible (lower part) features. 


\section{MATHEMATICAL TREATMENT OF INFINITY VIA THE ALEXANDER HORN}

Concerning AHS and the more general problem of the number of links in knots, linking numbers $\lambda$ (sum of the number of crossing points in a knot) as well as enhanced linking numbers have been introduced (Livingston 2003). A link is a collection of closed curves in 3D space without self-intersections. A link with one component is called a knot. Examples of links with 2 components are shown in Figure 3. In the study of knots (especially AHS), linking numbers can become unbounded (approaching infinite numbers of crossing points) rather quickly. For example, a twocomponent linking number is denoted by $\lambda(K, L)$, which ranges over the set of integers $2,3, \ldots, \infty$.

Also, AHS allows the introduction of geometric computable solutions to the problem of infinity. The basic approach is to rewrite metrics defined in terms of points at infinity with tractable, computable metrics that sidestep infinite planes and points at infinity. This permits to assess conformable infinity at computable points near, but not at, ideal points. In other words, ideal points on the infinite plane are replaced with non-ideal points that are computable, because they intersect sheets of infinite places. Here we provide an example from physics. The Minkowski spacetime metric in polar coordinates is $g=\mathrm{d} t^{2}-\mathrm{d} r^{2}-r^{2} \mathrm{~d} \sigma \tau^{2}$, where $\mathrm{d} \sigma \iota^{2}$ is the metric of the unit sphere. To achieve conformal rescaling of $g$, the null coordinates $u=1-r$ and $v=l-r$ are introduced, to obtain $g=\mathrm{d} u \mathrm{~d} v-1 / 4(v-u)^{2} \mathrm{~d} \sigma L^{2}$. The coordinates $u$ and $v$ range over the complete real line, provided $v-u \geq 0$. This infinite range is compacted, using $u=$ $\tan (U), v=\tan (V)$, and new null coordinates ranging over $(-\pi / 2,+\pi / 2)$ resulting in $g=\left[1-\left(4 \cos ^{2} U \cos ^{2} V\right)\right] \times\{4 \mathrm{~d} U \mathrm{~d} V-$ $\left.\sin ^{2}(V-U) \mathrm{d} \sigma L^{2}\right\}$, which is not defined at $U= \pm \pi / 2, V= \pm \pi / 2$. To solve this problem, a new metric is extracted from $g$, namely, $g_{\text {new }}=4 \mathrm{~d} U \mathrm{~d} V-\sin ^{2}(V-U) \mathrm{d} \sigma L^{2} . g_{\text {new }}$ is the tractable metric of the Einstein cylinder, which means that Minkowski's space is now conformably embedded into the Einstein cylinder. [51]
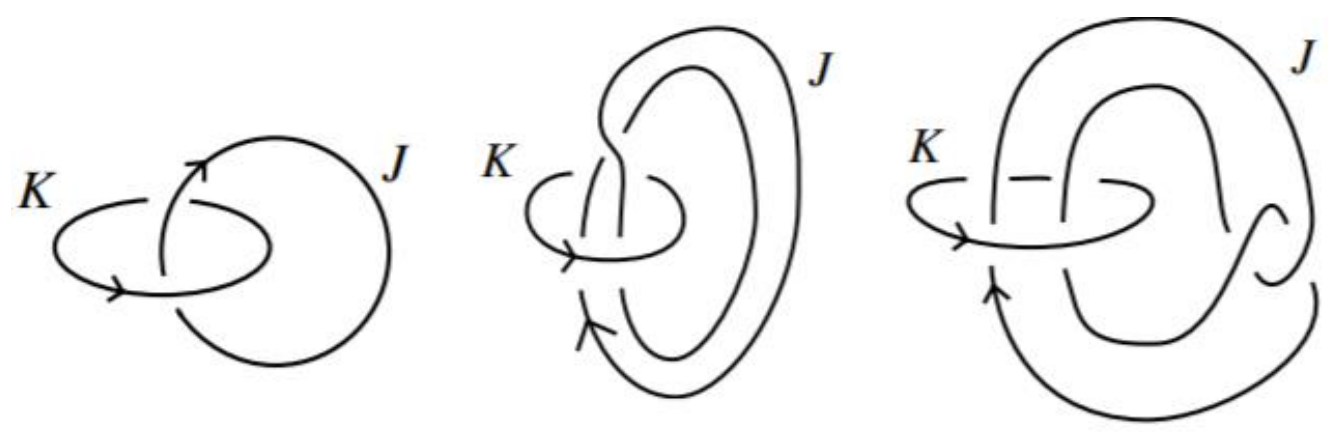

Figure 3. Examples of 2-component links. From: Livingston 2003.

\section{ALEXANDER TORUS IN THE STUDY OF PHYSICAL CONTINUUM: AN OPERATIONAL EXAMPLE}

Linear dynamics of physical and biological systems can be described in terms of trajectories taking place inside manageable donut-like manifolds equipped with genus one. However, widespread nonlinear dynamics might take place, such as paths at the edge of chaos, power law and free scale phenomena, systems' bifurcations, iterative maps, ordinary differential equations, Bayesian issues, temperature chaos detected in four-dimensional Edwards-Anderson models with Gaussian disorder to low temperatures (Wang et al., 2018). Here we ask: if we consider linear dynamics as indivisible, and non-linear chaotic dynamics as continuous, is it feasible to build a single donut-like phase space where both these dynamics might be mapped? The answer is positive, if we consider AHS. Both the linear and nonlinear paths can be arranged inside a phase space consisting of an AHS. This relatively simple general description of both the trajectories as taking place inside horned torus-shaped manifolds allows to standardize the assessment of a wide range of natural and artificial paths, from time series analysis to iterative maps, from population dynamics to temperature.

Countless linear biological trajectories, including nervous dynamics, can be tackled in terms of paths of different curvatures embedded in a three-dimensional phase space (Tozzi and Peters, 2019). As an example, here we provide a simulation of a linear flow that turns out chaotic when crossing a physical obstacle (Figure 3A). To map these dynamical paths to AHS, a standardized procedure can be performed that allows flow trajectories to be transported to the AHS surface (Figure 3B). Such projections can be achieved through available methodologies of vectorial and tensorial transport, such as Ehresmann connections (Tozzi et al., 2017) or parallel transport on Riemannian manifolds (Sengupta et al., 2017). It is noteworthy that different kinds of nonlinear paths can be assessed through this procedure: to make an example, Figure 3C illustrates the case of two mirror logistic plots embedded in an AHS. In sum, when we place physical or biological observables on the surface of AHS, we achieve the description of their different trajectories inside a single, peculiar donut-like phase space. 

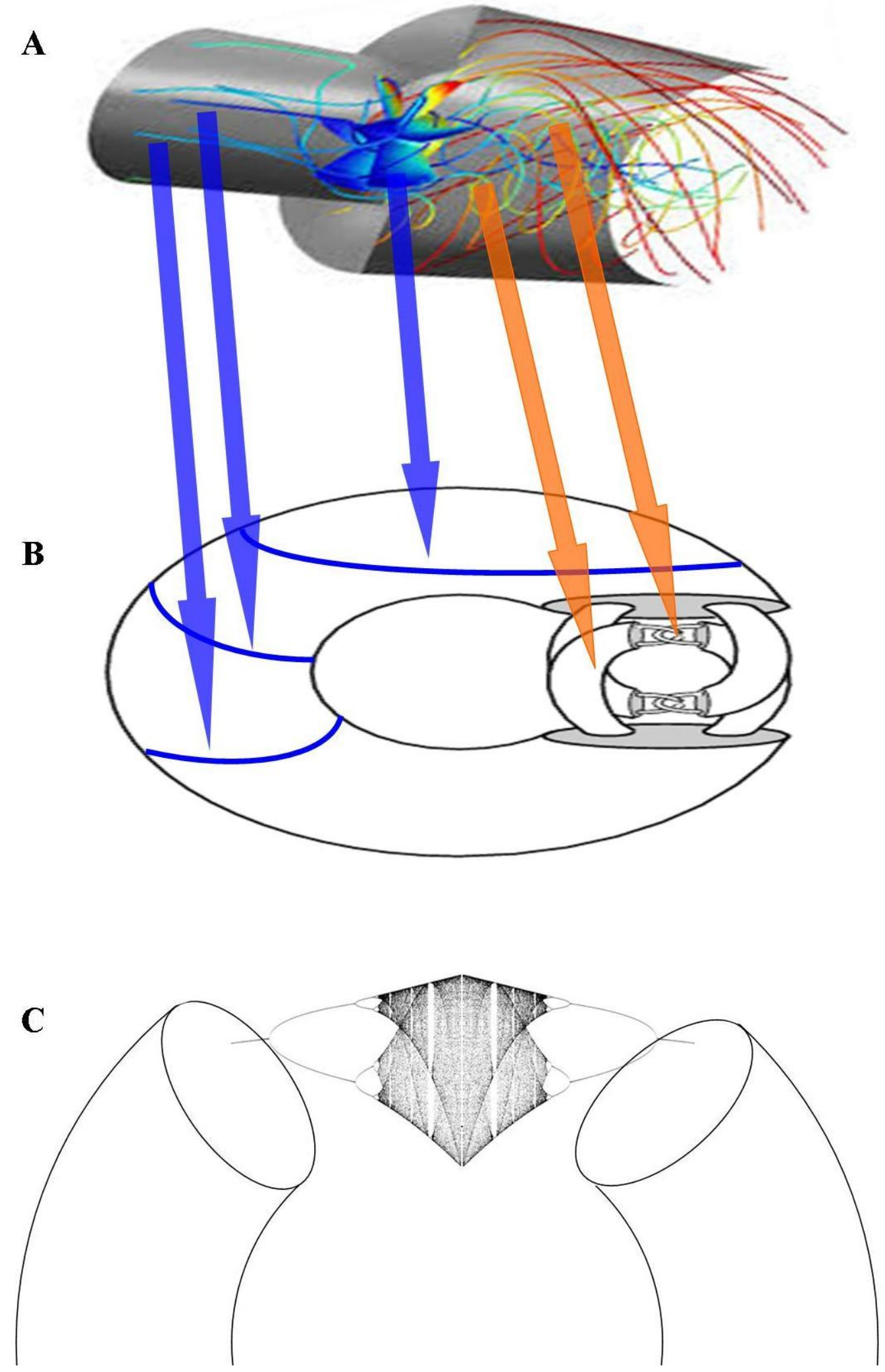

Figure 3. Mapping of real systems flows to AHS. Widespread physical and biological linear paths (Figure 3A) can be described in terms of trajectories taking place the toroidal side of AHS (blue arrows and curved lines in Figure 3B). In turn, non-linear trajectories can be projected to the branched section of the HAS (red arrows). Modified from: CFD Moduule. https://www.technic.com.au/products/cfd/ (Retrieved 2018). Figure 3C. One of the feasible modifications of the Alexander horned sphere. Here two opposite logistic plots are inserted into the branched section of the horned torus. 


\section{CONCLUSION: SOLVING INFINITY}

It is assumed by physicists, due to pragmatic issues, that no measurable quantity or event has infinite values. Indeed, any physical theory needs to provide operational tools that correspond to, or at least approximate, reality (e.g., Iyer and Petters, 2007). Consider, for example, if any object of infinite gravitational mass were to exist, any use of the formula in order to quantify the gravitational force would lead to a useless infinite result. The formula would be useful neither to compute the force between two objects of finite mass, nor to compute their motions. Sometimes an infinite result of a physical quantity may mean that the theory being used is approaching the point where it fails.

To solve the problem of the meaningless occurrence of infinity in physical equations, in particular the ones concerning thermal issues, a novel geometrically-framed conceptual framework has been proposed here, based on a geometric perspective. The mapping of real systems' paths to AHS permits us to achieve manageable representations of countless biological trajectories inside peculiar donut-like phase spaces. This allows to simplify the measurement procedures, because paths on toruses have been very well studied in several fields, such as in modular wavelets, admissibility and frame conditions, lifting admissible functions, transformations and dilations, reconstruction formulas, natural tensor products, required Lie groups and extra modular groups (Tozzi et al., 2018; Calixto et al., 2015; Wang et al., 2015). Furthemore, a conceptual leap is provided: in this novel framework, a continuous monodimensional line becomes a collection of countless bidimensional lines that superimpose in quantifiable knots and bifurcations.

\section{REFERENCES}

1) Alexander, J. W. "An Example of a Simply Connected Surface Bounding a Region Which Is Not Simply Connected." Proc. N. A. S. 10, 8-10, 1924.

2) Aristotle (1980). Physics, 2 volumes, trans. Cornford and Wickstead. Loeb Classical Library, Cambridge, MA: Harvard University Press and Heinemann.

3) Asselmeyer-Maluga T. 2018. Hyperbolic groups, 4-manifolds and Quantum Gravity. arXiv:1811.04464.

4) Bell, J. L. (2005) The Continuous and the Infinitesimal in Mathematics and Philosophy, Milan: Polimetrica S.A

5) Bergmann, 1989. Quantum gravity at spatial infinity. General Relativity and Gravitation 21, no. 3, $271-278$.

6) Bradwardine T. 1328-1335. Tractatus de continu. Trans by Murdoch JE. Quoted in Edward Grant ed., a source book in Medieval science. Cambridge, Massachusetts: Harvard University Press, 1974.

7) Bridges, D.(1999). "Constructive mathematics: a foundation for computable analysis," Theoretical Computer Science, 219: 95-109.

8) Calixto M, Guerrero J, Roşca D. 2015. Wavelet transform on the torus: A group theoretical approach. Applied and Computational Harmonic Analysis, 38 (1): 32-49. https://doi.org/10.1016/j.acha.2014.03.001.

9) Cantor, G., (1961). Contributions to the Founding of the Theory of Transfinite Numbers, New York: Dover.

10) de Cusa N. 1440. De docta ignorantia. English translation in Bond, H. Lawrence (ed.), Nicholas of Cusa: Selected Spiritual Writings, Classics of Western Spirituality. New York: Paulist Press, 1997.

11) Dedekind, R. (1963). Essays on the Theory of Numbers, New York: Dover.

12) Ehresmann C. 1950. Les connexion s infinitésimales dans un espace fibré différentiable, Colloque de Toplogie, Bruxelles, pp. 29-55

13) Frauendiener, 2000. Conformal infinity. Living Reviews in Relativity, http://www.springer.com/ us/livingreviews/articles/volume3/2000-4frauendiener

14) Frankel T. 2011. The Geometry of Physics: An Introduction. Cambridge University Press; IIIrd Ed. ISBN13: $978-1107602601$

15) Iyer and Petters, 2007. Light's bending angle due to black holes: from the photon sphere to infinity. Gen. Relativ. Grav. 39, 1563-1582.

16) Ji Z, Natarajan A, Vidick T, Wright J, Yuen H. 2020. MIP*=RE. arXiv:2001.04383.

17) Langevin P. 1908. Sur la théorie du movement brownien. C. R. Acad. Sci. Paris. 146: 530-533.

18) Lawvere, F. W. (1980). "Toward the description in a smooth topos of the dynamically possible motions and deformations of a continuous body," Cahiers de Top. et Géom. Diff., 21: 377-92.

19) Levi-Civita T. 1917. Nozione di parallelismo in una varietà qualunque e conseguente specificazione geometrica della curvatura Riemanniana. Rend. Circ. Mat. Palermo, 42: 73-205, doi:10.1007/bf03014898

20) Livingston C, Enhanced Linking Numbers, The American Mathematical Monthly (2003), 110:5, 361-385, DOI: $10.1080 / 00029890.2003 .11919975$

21) Mandelstam L, Tamm I. 1945. The uncertainty relation between energy and time in nonrelativistic quantum mechanics. J. Phys. (USSR) 9, 249-254.

22) Poincaré. H. (1946). Foundations of Science, trans. G. Halsted, New York: Science Press. 
23) Robinson, A. (1996). Non-Standard Analysis, Princeton: Princeton University Press.

24) Sengupta B, Tozzi A, Coray GK, Douglas PK, Friston KJ. 2016. Towards a Neuronal Gauge Theory. PLOS Biology 14 (3): e1002400. doi:10.1371/journal.pbio.1002400.

25) 'tHooft G. 1971. Renormalizable Lagrangians for massive Yang-Mills fields. Nuclear Physics B. 35, $167-188$.

26) Tozzi A, Peters JF, Fingelkurts AA, Fingelkurts AA, Marijuán PC. 2017. Topodynamics of metastable brains. Physics of Life Reviews, 21, 1-20. http://dx.doi.org/10.1016/j.plrev.2017.03.001.

27) Tozzi A, Peters JF, Fingelkurts A, Fingelkurts A, Perlovsky L. 2018. Syntax meets semantics during brain logical computations. ProgrBiophys Mol Biol, 140 : https://doi.org/10.1016/j.pbiomolbio.2018.05.010.

28) Tozzi A, Peters JF. 2019 Points and lines inside our brains. Cognitive Neurodynamics. DOI: 10.1007/s11571-019-09539-8.

29) Tozzi A, Peters JF. 2019. Points and lines inside our brains. Cognitive Neurodynamics, 13(5): $417-428$. DOI: $10.1007 / \mathrm{s} 11571-019-09539-8$.

30) Wang BQ, Wang Gm Zhou X-H. 2015. A local wavelet transform on the torus T2. International Journal of Wavelets, Multiresolution and Information Processing. $13 \quad$ (04): 1550027. https://doi.org/10.1142/S0219691315500277.

31) Wang W, Wallin M, Lidmar J. 2018. Chaotic temperature and bond dependence of four-dimensional Gaussian spin glasses with partial thermal boundary conditions. Phys. Rev. E 98, 062122. 